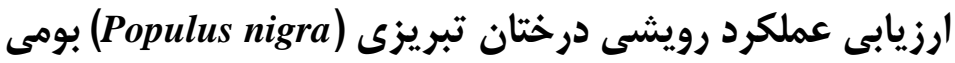

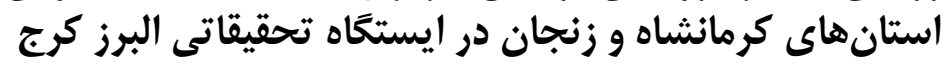

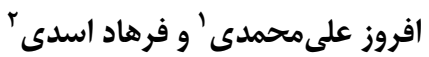

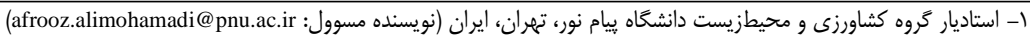

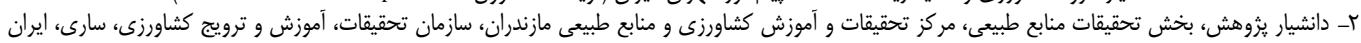
9V/V/A تاريخ يذيرش

صفحd: •1 19 9V/\&/19: تاريخ دريافت

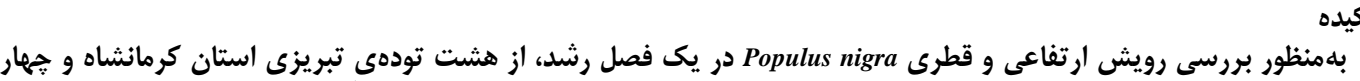

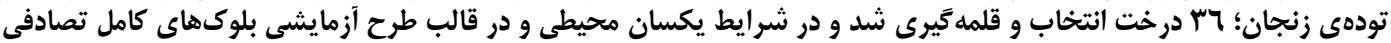

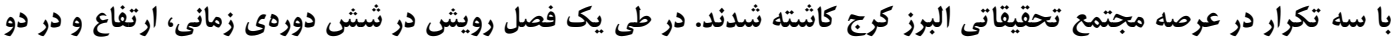

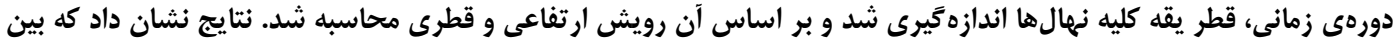

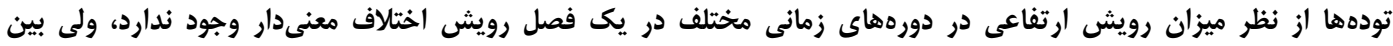

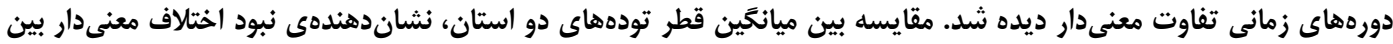

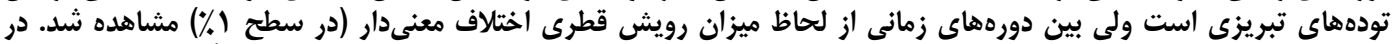

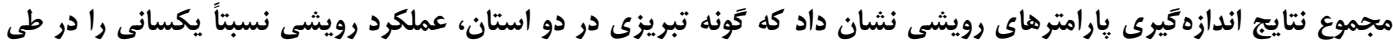
يك فصل رشد داشتند.

وازههاى كليدى: رويش ارتفاعى، رويش قطرى، Populus nigra، تبريزىهاى كرمانشاه و تبريزىهاى زنجان

عملكرد رويشى، ارزيابى كيفى تودهها، برآورد وراثتيذيرى

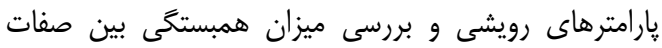

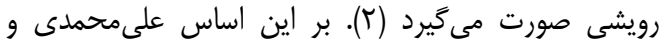

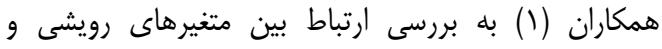
مورفولوزيكى دو كَونه

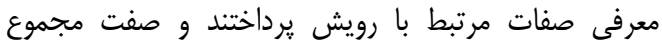

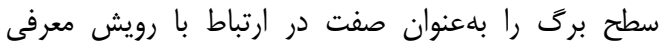

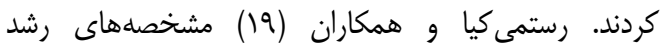

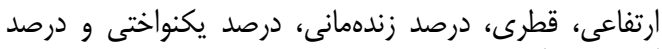

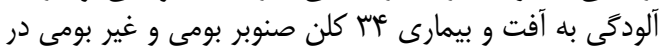

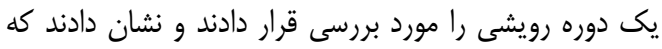

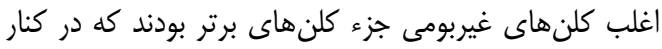

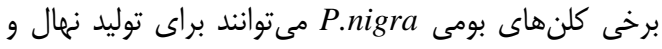

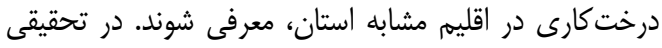

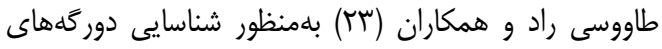

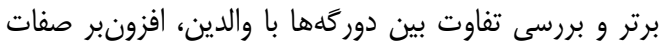

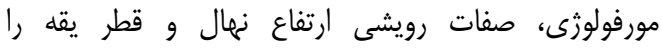

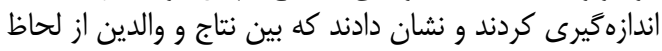

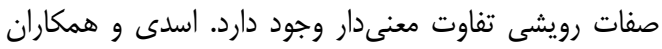

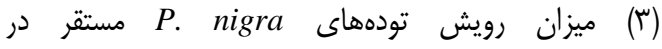
صنوبر كارىهاى مردمى (سنتى) استان كرمانشاه، بلهعنوان

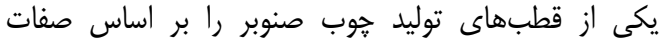

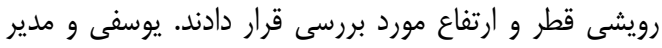

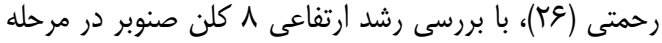

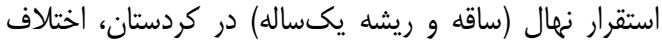

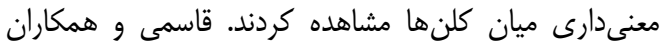

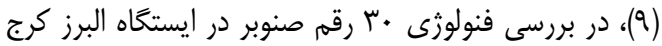

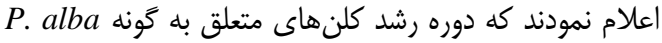

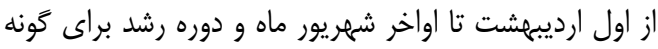

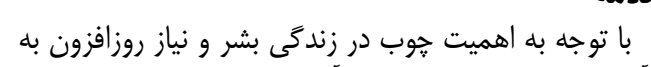

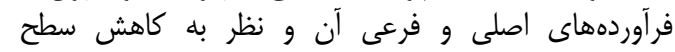

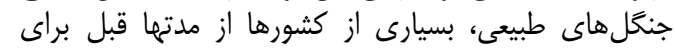

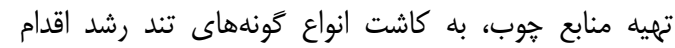

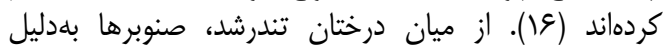

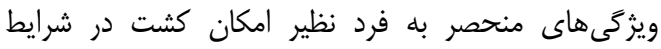

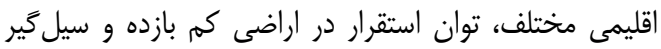

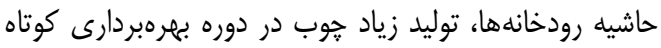
مدت، سهولت تكثير رويشى، امكان كشت تورام تورئ باد با محصولات

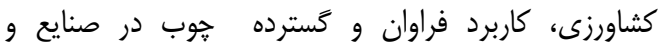

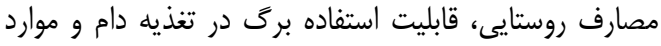

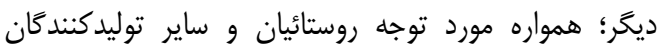

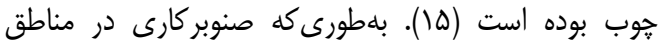

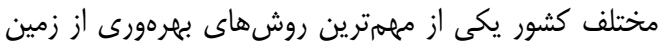

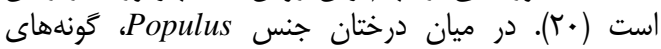
تبريزى (Pigra)

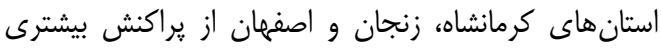

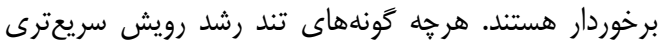

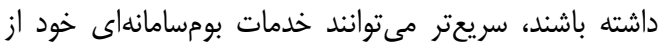

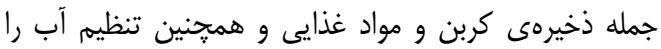

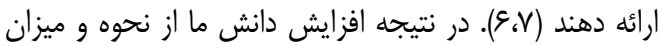

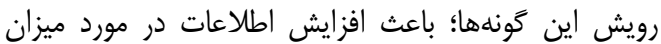

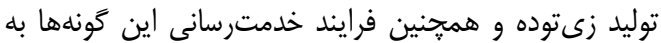

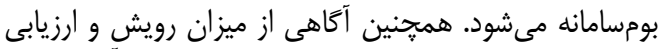

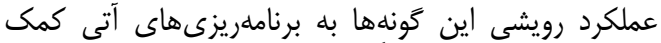

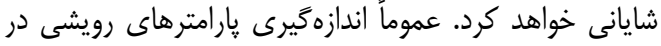

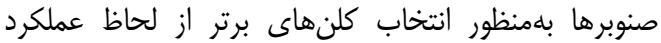
رويشى، مقايسه تودهها، كَونها و هيبر هايدهاى يرتي مختلف از از نظر 
ᄉ)

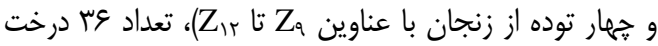

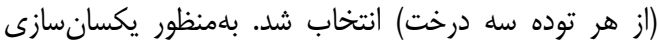

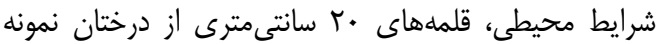

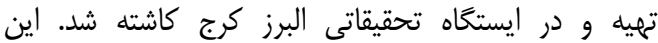

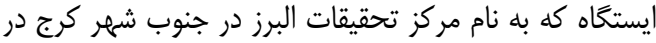

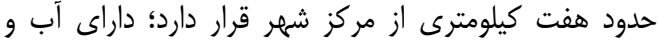

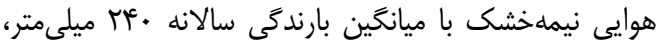

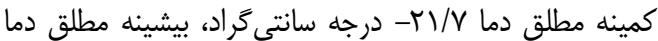
أF درجه سانتى

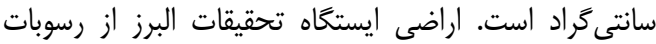

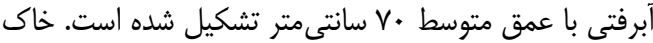

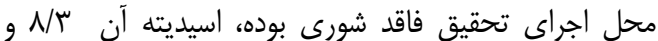

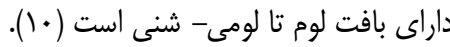

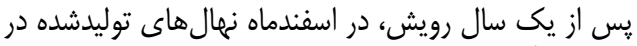

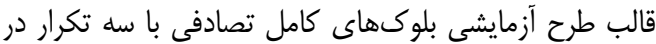

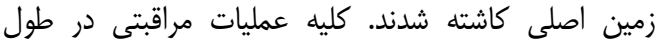

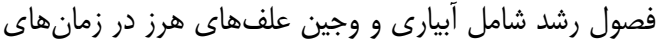

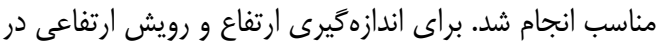

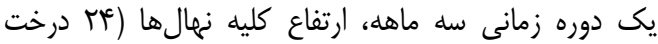

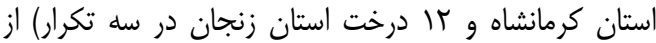

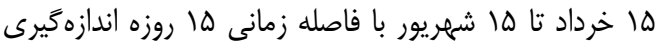

سيس براى اندازهگيرى رويش ارتفاعى مربوط به هر مقطع

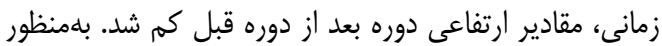

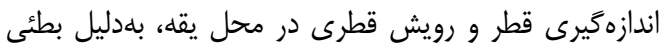

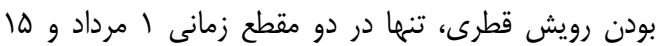

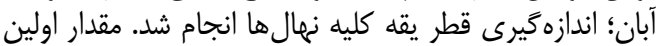

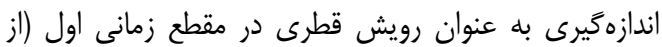

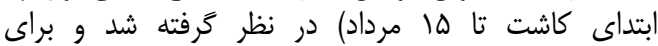

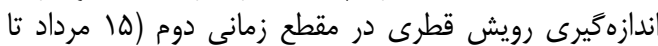

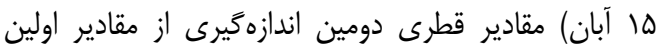

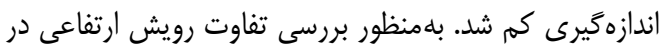

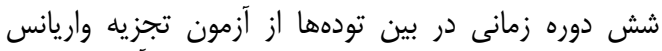

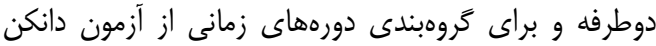

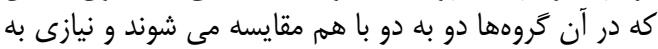

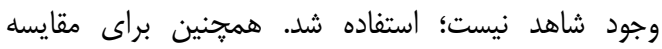

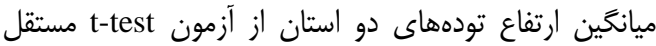

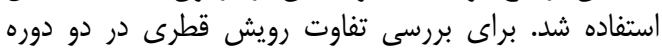

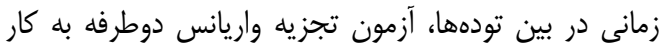

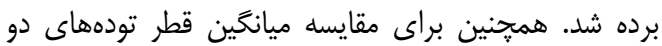

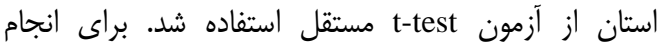

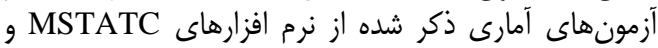
SPSS (v21)

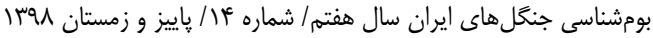

P. nigra

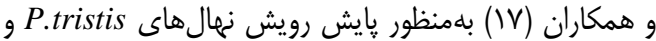
P.euramericana

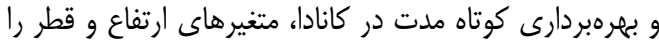

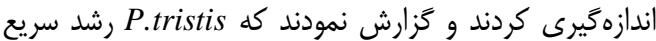

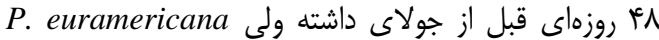

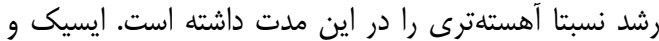

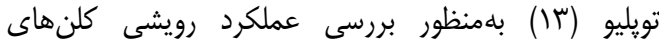

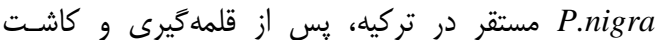

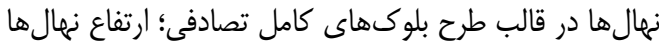

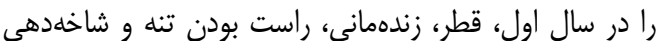

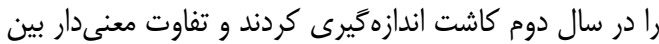

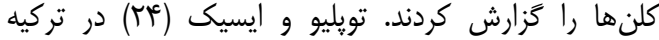

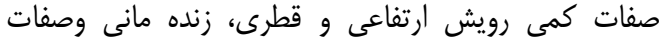

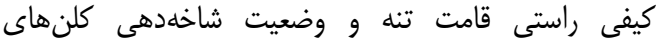

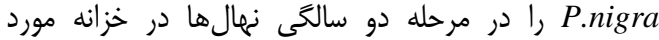

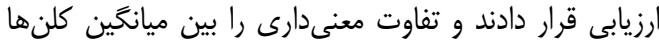
از نظر صفات فوق ارائه دادند.

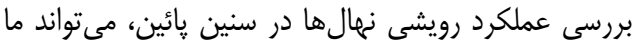

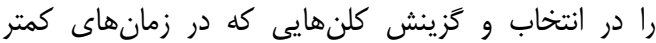

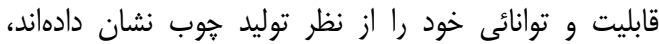

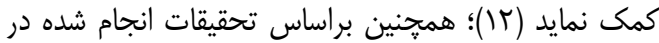

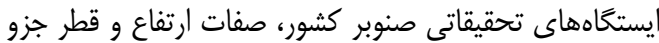

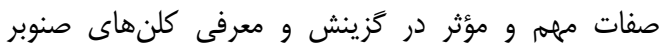

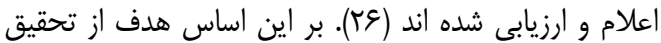

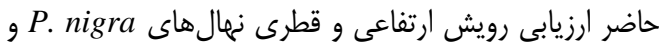

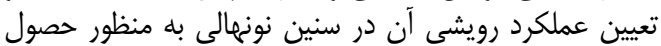

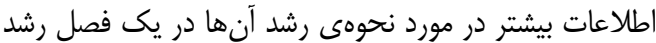

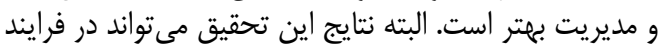
انتخاب اوليه ارقام يربازده و زودبازده به كار آيد.

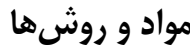

از تودههاى صنوبر كارى شده استان كرمانشاه، هشت تودي

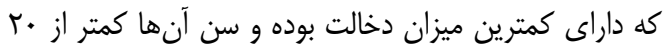

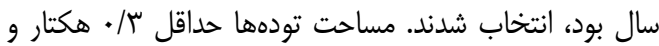

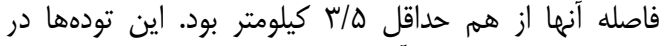

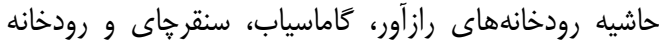

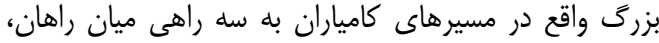

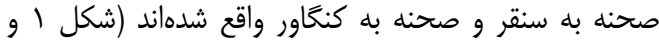

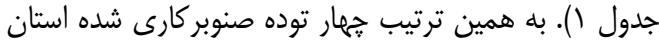

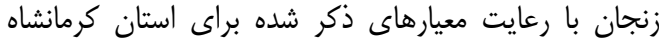

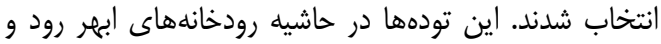

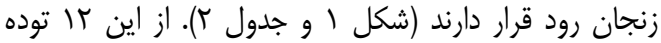

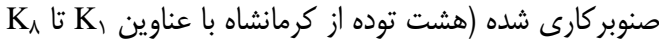




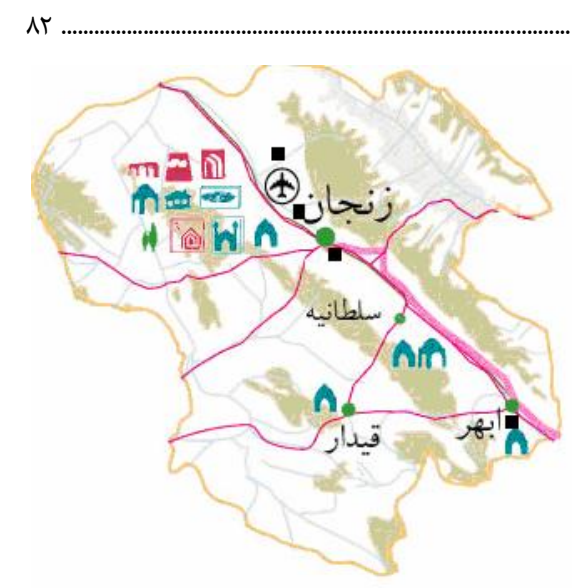

ارزيابى عملكرد رويشى درختان تبريزى (Populus nigra) بومى استانهاى كرمانشاه و زنجان .

شكل 1- موقعيت تودههاى مورد بررسى (مربعهاى سياه) در سطح دو استان

Figure 1. Locations of studied stands (black squares) in the two provinces

Table 1. Characteristics and locations of studied stands in Kermanshah province

\begin{tabular}{|c|c|c|c|c|}
\hline توده & 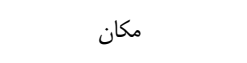 & فاصله مركز توده از بستر & ارتفاع از سطح دريا & $\begin{array}{l}\text { مساحت } \\
m^{r}\end{array}$ \\
\hline $\mathrm{K}_{1}$ & مسير كامياران به ميانراهان & $r \cdot$ & ITTQ & $11 \%+$. \\
\hline $\mathrm{K}_{\mathrm{r}}$ & qa كيلومترى توده يك & Q. & $1 f+$. & $11 \cdot 1$. \\
\hline $\mathrm{K}_{r}$ & مسير صحنه به سنقر & $1 \ldots$ & ivr. & $1.1 \%$ \\
\hline $\mathrm{K}_{\mathrm{r}}$ & ه/ه كيلومترى توده سه & r. & $19 \Lambda$. & १QT. \\
\hline $\mathrm{K}_{\Delta}$ & ه/ه كيلومترى توده جهار & $\Delta$. & 199. & $111 .$. \\
\hline $\mathrm{K}_{\varphi}$ & ז T كيلومترى توده ضينج & $1 .$. & $\wedge \Delta$. & 1199. \\
\hline $\mathrm{K}_{\mathrm{r}}$ & . ا كيلومترى توده دو. & f. & 149. & s.F. \\
\hline $\mathrm{K}_{\wedge}$ & كنغاور & 1. & 149. & $81 .$. \\
\hline
\end{tabular}

جدول r- مشخصات و موقعيت تودههاى مورد بررسى در استان زنجان Table 2. Characteristics and locations of studied stands in Zanjan province

\begin{tabular}{|c|c|c|c|c|}
\hline 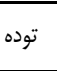 & 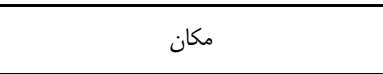 & $\begin{array}{c}\text { فاصله مركز توده از } \\
\text { m بس }\end{array}$ & ارتفاع از سطح دريا & مساحت \\
\hline$Z_{a}$ & جنوب ابهر & $1 \ldots$ & $10 T$ & $1 \ldots$ \\
\hline$Z_{1}$ & •ا كيلومترى زنجان در سمت جنوب شرقى. & $\Delta \cdot$ & 191. & $r \cdots$ \\
\hline$Z_{11}$ & شمال على آباد & f. & IQF. & a... \\
\hline$Z_{i r}$ & ه/ •ا كيلومتر بعد از توده ل & r. & $|Q|$. & $8 \ldots$ \\
\hline
\end{tabular}

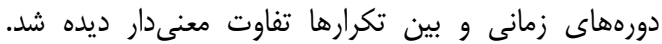

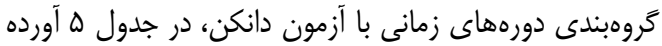

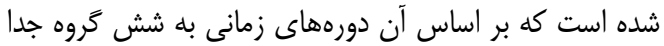

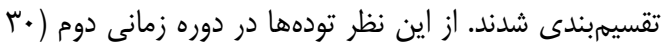

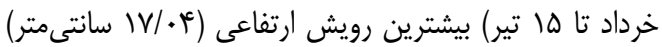

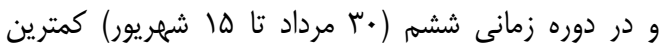

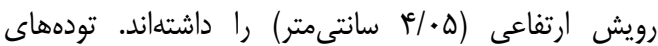

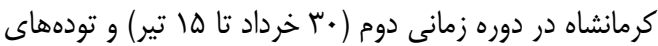

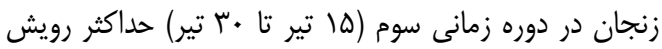
ارتفاعى را داشتند (شكل در زانى).

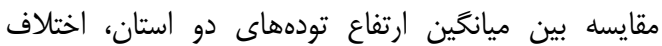

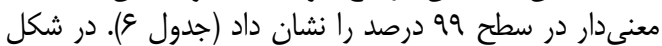

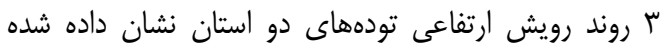

\section{نتايج و بحث

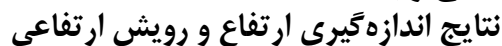

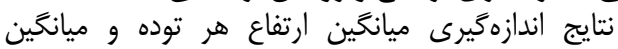

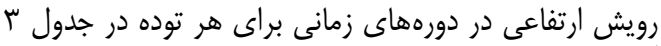

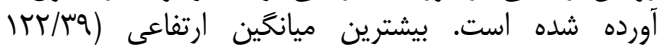

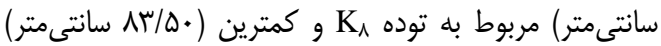

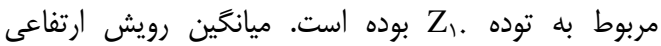

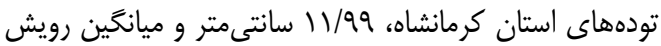

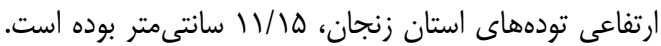

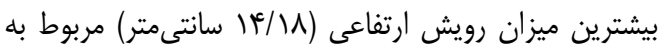

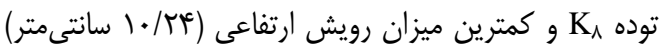

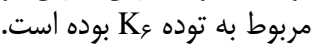

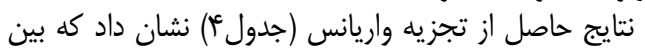

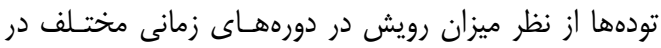

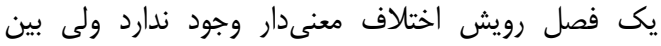




$$
\text { ^ז... }
$$

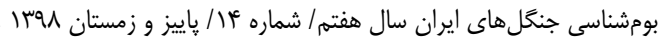

$$
\text { جدول ب- ميانگين ارتفاع تودهها و ميانگين رويش ارتفاعى (سانتىمتر) تودهها در دورههاى زمانى }
$$

\begin{tabular}{|c|c|c|c|c|c|c|c|c|}
\hline \multirow[b]{2}{*}{ توده } & \multirow[b]{2}{*}{$\begin{array}{c}\text { ميانكَين ارتفاع cm } \\
\text { cm }\end{array}$} & \multicolumn{7}{|c|}{ ميانكين رويش ارتفاعى در دورههاى زمانى Cm } \\
\hline & & 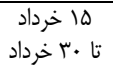 & ها لخرداد تا تا & 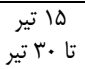 & تا هامرداد & 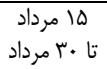 & تا ها شمر مراد & ميانگين براى هر \\
\hline $\mathrm{K}_{1}$ & $1 \cdot N / 1$ & Ir/AF & $r / / v \wedge$ & $10 / \lambda \mu$ & $1 \% / \lambda$ & $\Delta / \Delta \Lambda$ & $f / V F$ & $|r / r|$ \\
\hline $\mathrm{K}_{\mathrm{r}}$ & $1 . \Delta / V$. & $\mid f / \Lambda \mu$ & $19 / .4$ & $\mid Q / \Delta \Lambda$ & $11 / r$. & S/IT & $r / \Delta \Lambda$ & $11 / \Delta S$ \\
\hline $\mathrm{K}_{\mathrm{r}}$ & או/../ & IT/GT & W/GT & $15 / 19$ & $\mid r / 1$. & $\Delta / M$ & $r / q V$ & $1 \cdot / 1 \Delta$ \\
\hline$K_{f}$ & $11 \% / .9$ & $11 / \Delta S$ & $19 / 19$ & $1 F / V V$ & $|r / \Delta|$ & V/FT & $\Delta / \Delta \Lambda$ & $I T / N$ \\
\hline $\mathrm{K}_{\Delta}$ & ११/.। & $\mid r / F Y$ & $19 / 97$ & IV/AT & $|r / 4|$ & $9 / \pi F$ &.$/ 91$ & 11/9 \\
\hline $\mathrm{K}_{\varphi}$ & q & IT/Tr & $\mid 9 / \cdot V$ & $1 F / \Delta 9$ & 11/.r & $r / v \varepsilon$ & $1 / V^{e}$ & $1 . / \pi F$ \\
\hline $\mathrm{K}_{\mathrm{v}}$ & $1.0 / 99$ & $|r / \Delta|$ & $19 / \mu r$ & $1 \% / v 9$ & IT/NT & $1 \cdot / 94$ & $r / \Delta V$ & $11 / v 8$ \\
\hline $\mathrm{K}_{\wedge}$ & Q & $14 / 4 q$ & $r / \mathrm{r}$ & N/IT & $14 / \mathrm{Vq}$ & $9 / 41$ & s/ve & $\mid f / M$ \\
\hline$Z_{q}$ & $91 / 99$ & N/Fr & $11 / r q$ & $\mid f / \cdot 1$ & $14 / 19$ & $11 / 4 \varepsilon$ & $\Delta / \Delta)$ & $1 . / V 8$ \\
\hline$Z_{1}$. & $\Lambda \kappa / \Delta$. & N/Q1 & W/a & ID/IT & $\mid f / \cdot 1$ & $1 \cdot / 1$ & $r / \Delta 1$ & 1.199 \\
\hline$Z_{11}$ & $9 \% / \leftarrow \&$ & $1 . / 4$. & $\mid r / \cdot T^{4}$ & $\mid f / \cdot V$ & $1 \pi / v \wedge$ & $1.1 / 9$ & $r / \mu^{2}$ & $1 . / \mathrm{vA}$ \\
\hline$Z_{1 r}$ & 91/9V & $1 / 48$ & $1 \% / q 9$ & $19 / \mathrm{Vg}$ & I $8 / A V$ & $11 / \mp \wedge$ & $V / 99$ & g \\
\hline
\end{tabular}

Table 3. Mean height and mean height growth of stands in different time periods

جدول عأ- مقادير آمارههاى حاصل از مقايسه ميانخينهاى رويش ارتفاعى با تجزيه واريانس

\begin{tabular}{|c|c|c|c|}
\hline منبع تغييرات & درجه آزادى & ميانگين مربعات & F آماره F \\
\hline تكرار & $r$ & $1 \% q / 1 \mu$ & $N / \kappa^{*}{ }^{* *}$ \\
\hline توده & 11 & $r F / F$. & $1 / 4 \lambda^{\mathrm{ns}}$ \\
\hline زمان & $\Delta$ & $1 \cdot 9 / V^{c}$ & $r q / / V^{* *}$ \\
\hline توده×زمان & $\Delta Q$ & $I V / \backslash \Lambda$ & $1 / \cdot e^{\mathrm{ns}}$ \\
\hline خطا & IFT & $\mid s / 4 V$ & --- \\
\hline
\end{tabular}

Table 4. Statistics of mean height growth comparison based on Analysis of Variance

جدول ه- ميانگين و درصد رويش ارتفاعى در شش دوره زمانى و گروهبندى دورههاى زمانى بر اساس ميانخين رويش ارتفاعى

Table 5. Mean and percentage of height growth in six time periods and grouping time periods based on mean height

\begin{tabular}{|c|c|c|c|c|}
\hline دوره & فاصله زمانى & كروهبندى & درصد رويش ارتفاعى & ميانخين رويش ارتفاعى \\
\hline اول & ها خرداد تا • خ خرداد & $\mathrm{C}$ & IV & $11 / N \varepsilon$ \\
\hline دوم & • ب خرداد تا ها تير & A & rf & $\mathrm{IV} / \cdot \mathrm{r}$ \\
\hline سوم & ها تير تا •r تير & $\mathrm{AB}$ & tr & $|\Delta / T|$ \\
\hline جهارم & • ץ تير تا ها مرداد & $\mathrm{BC}$ & 19 & $|r / \Delta|$ \\
\hline ينجم & ها مرداد تا • ب مرداد & $\mathrm{D}$ & it & $N / \Delta V$ \\
\hline ششم & •r مرداد تا ها شهريور. & $\mathrm{E}$ & $\varepsilon$ & $F / \cdot \Delta$ \\
\hline
\end{tabular}
growth

جدول צ- مقادير آمارههاى حاصل از آزمون t براى مقايسه ميانخين ارتفاع تودههاى دو استان

\begin{tabular}{|c|c|c|c|}
\hline منبع تغييرات & درجه آزادى & انحر اف معيار & t t t to to \\
\hline استان & 1. & $r / \Lambda)$ & $\Gamma / \Upsilon \Lambda * *$ \\
\hline
\end{tabular}
Table 6. Statistics of mean height comparison of two provinces stands based on t-test 

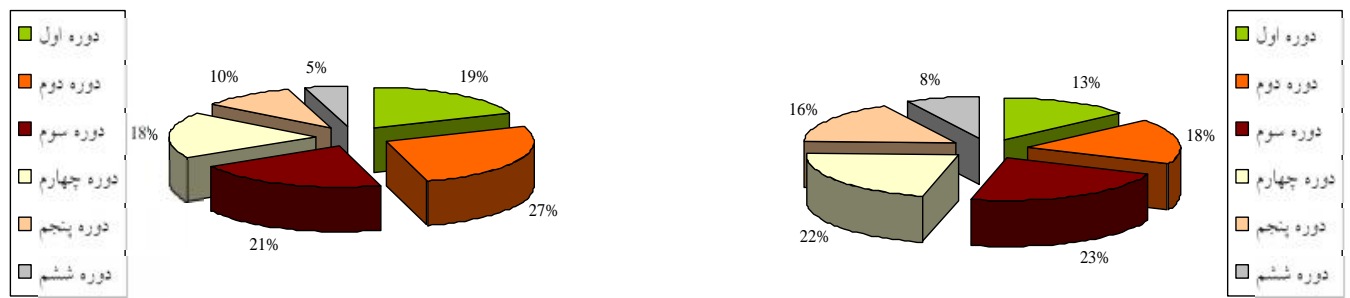

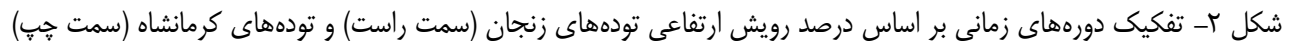

Figure 2. Splitting of time periods based on percentages of height growth of Zanjan stands (right) and Kermanshah stands (left)

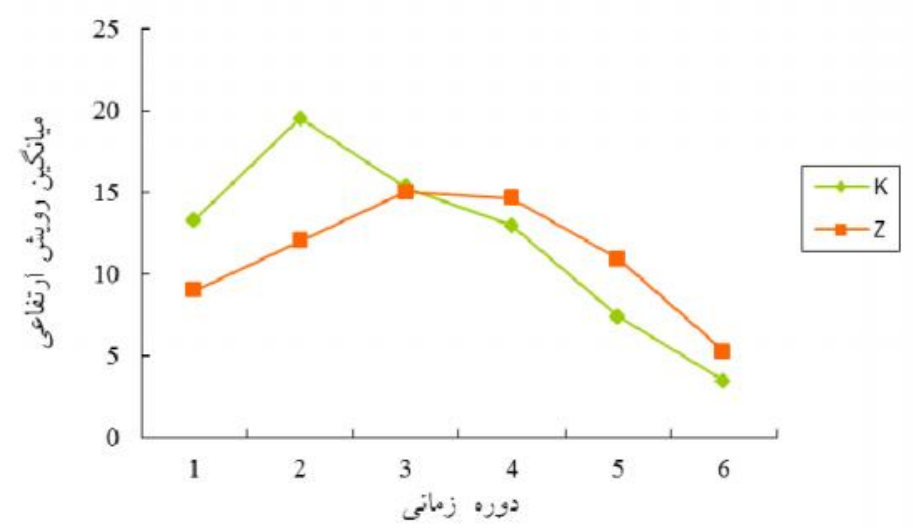

شكل بـ- روند رويش ارتفاعى تودههاى استان كرمانشاه (خط سبز) و زنجان (خط قرمز) در شش دوره زمانى

Figure 3. Height growth trends of Kermanshah (Green Line) and Zanjan (Red Line) stands in six time periods

معنى دار ديده شد (جدول 1). در شكل ه م تفكيك دورههاى

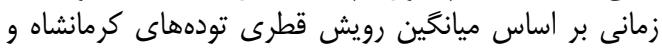

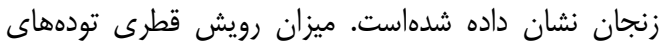

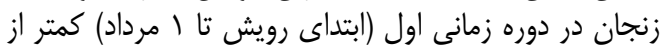

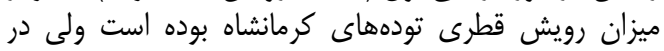

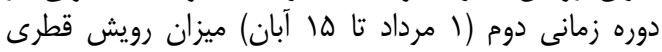
تودههاى زنجان بيشتر از تودههاى كرمانشاه بودان بوده است.

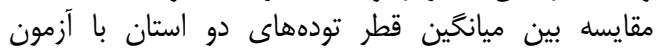

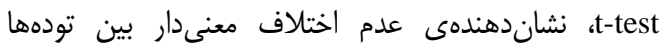

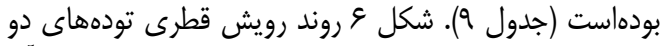

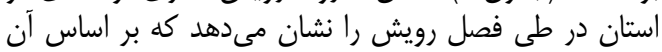

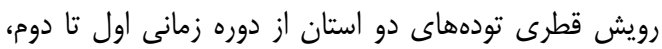
روند كاهشى را طى كرده كه اين رونان روند در بين تودههاى دو دواني

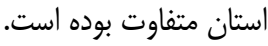

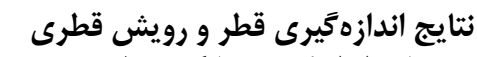

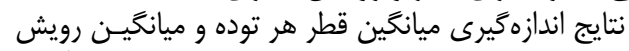

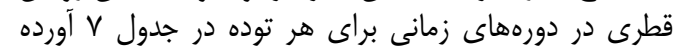

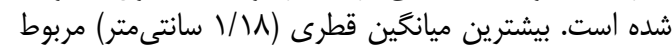

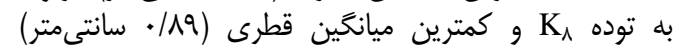

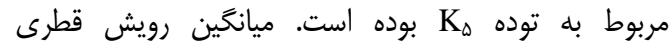

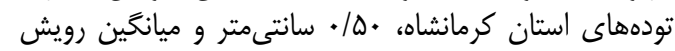

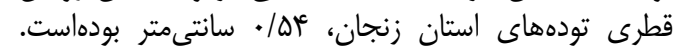

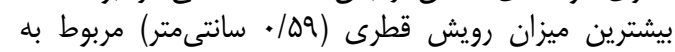

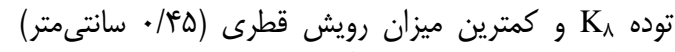
مربوط به توده بr و

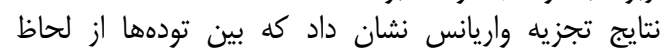

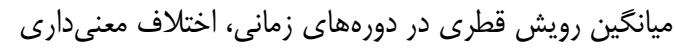

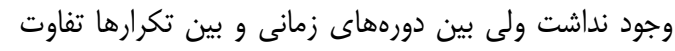


جدول V- - ميانخين قطر و ميانكين رويش قطرى (سانتىمتر) تودها در دورههاى زمانى Table 7. Mean diameter and mean diameter growth of stands in different time periods

\begin{tabular}{|c|c|c|c|c|}
\hline \multirow[b]{2}{*}{ توده } & \multirow[b]{2}{*}{ ميانكين قطر } & \multicolumn{3}{|c|}{ 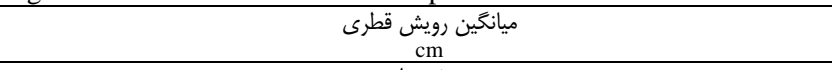 } \\
\hline & & ابتداى رويش تا ا مرداد & تا ها آبان & ميانكين براى هر توده \\
\hline $\mathrm{K}_{1}$ & $1 / \cdot f$ & $\cdot / A V$ & $\cdot / \mathrm{V}$ & $\cdot / \Delta T$ \\
\hline $\mathrm{K}_{\mathrm{r}}$ & $1 / \cdot 1$ & .119 &.$/ 1 r$ & $\cdot / \omega$ \\
\hline $\mathrm{K}_{r}$ &.$/ 91$ & $\cdot / \Lambda$ & .11 &.$/ \$ a$ \\
\hline $\mathrm{K}_{\mathrm{r}}$ & $1 / . r$ &.$/ 9$ &.$/ 1 f$ & . $/ \Delta T$ \\
\hline $\mathrm{K}_{\Delta}$ &.$/ 19$ & $\cdot / \Lambda$ & .11 &.$/ \$ \Delta$ \\
\hline $\mathrm{K}_{\varphi}$ &.$/ 9 T$ & $\cdot|\wedge|$ &.$/ 11$ &.$/ 48$ \\
\hline $\mathrm{K}_{\mathrm{v}}$ & ( & . & .11 &.$/ 48$ \\
\hline $\mathrm{K}_{\wedge}$ & $1 / M$ &.$/ 99$ &.$/ 19$ & .109 \\
\hline$Z_{q}$ & $1 / \cdot 1$ & $\cdot / N$ & ه און. & $.10 \%$ \\
\hline$Z_{1}$. &.$/ 94$ & $\cdot / V^{4}$ &.$/ T \Delta$ & $\cdot / \Delta$ \\
\hline$Z_{11}$ & $1 / \cdot r$ & $\cdot / v 9$ & ع & $\cdot / \Delta V$ \\
\hline$Z_{i r}$ &.$/ 91$ &.$/ N$ &.$/ \mu f$ & . \\
\hline
\end{tabular}

جدول ^ــ مقادير آمارههاى حاصل از مقايسه ميانخينهاى رويش قطرى با تجزيه واريانس Table 8. Statistics of mean diameter growth comparison based on Analysis of Variance

\begin{tabular}{|c|c|c|c|}
\hline منبع تغييرات & درجه آزادى & ميانخين مربعات & F آماره F \\
\hline تكرار & $r$ & $\cdot / 1 \cdot \Delta$ & $9 / \Delta f^{* *}$ \\
\hline توده & 11 & .1 .14 & $1 / \pi e^{n s}$ \\
\hline زمان & 1 & $V / \cdot \Delta$ & $94.1 . .^{* *}$ \\
\hline توده×زمان & 11 & גץ./. & $r / \subset q^{* *}$ \\
\hline خطا & is & $.1 \cdot 11$ & ---- \\
\hline
\end{tabular}

جدول q- مقادير آمارههاى حاصل از آزمون t براى مقايسه ميانغين قطر تودههاى دو استان Table 9. Statistics of mean diameter comparison of two provinces stands based on t-test

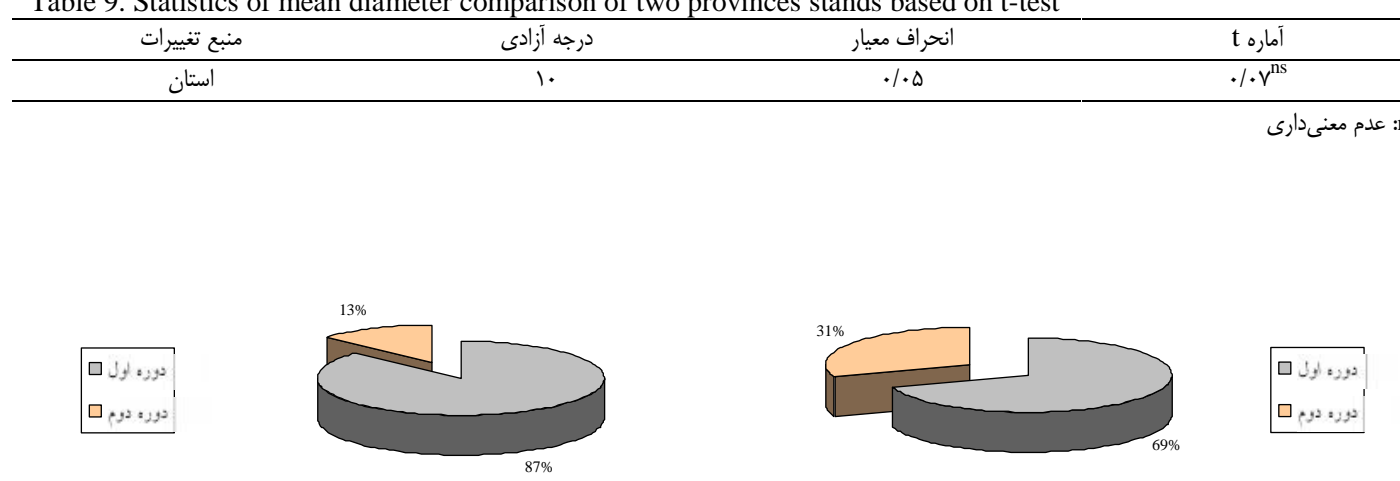

شكل هـ تفكيك دورههاى زمانى بر اساس درصد رويش قطرى تودههاى زنجان (سمت راست) و تودههاى كرمانشاه (سمت جٍ)

Figure 5. Splitting of time periods based on percentages of diameter growth of Zanjan stands (right) and Kermanshah stands (left) 


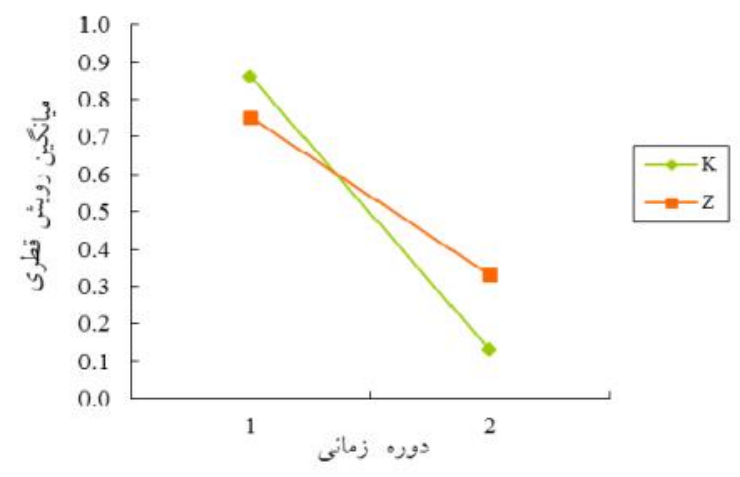

شكل צ- روند رويش قطرى تودههاى استان كرمانشاه (خط سبز) و زنجان (خط قرمز) در دو دوره زمانى

Figure 6. Diameter growth trends of Kermanshah (Green Line) and Zanjan (Red Line) stands in six time periods

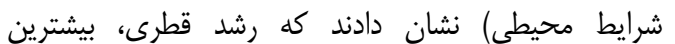

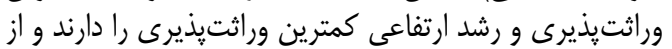

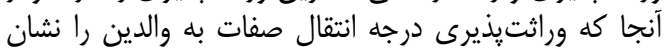

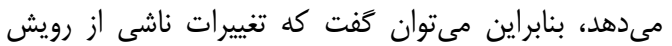

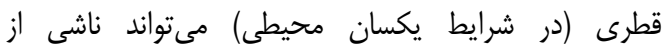

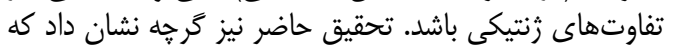

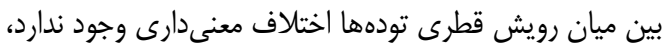

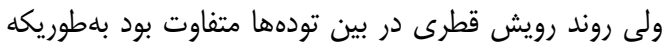

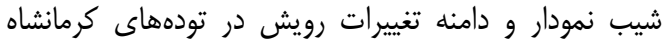

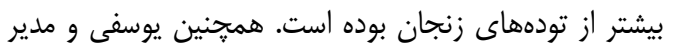

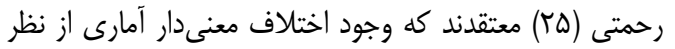

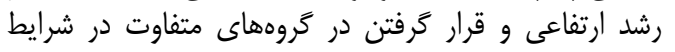

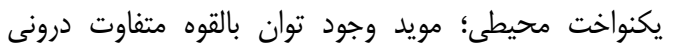

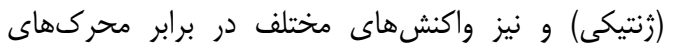

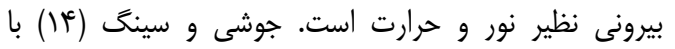

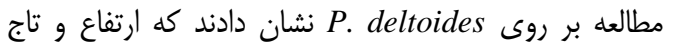
درختان به طور قابل ملاحظهاى وراثتيذير هستند و ور بازده زنتيكى اين صفات بسيار بالاست.

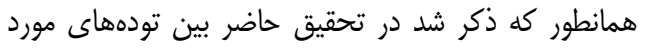

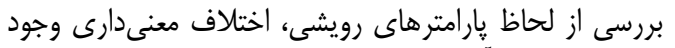

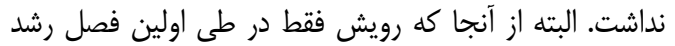

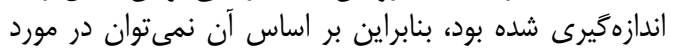

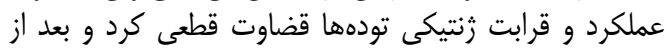

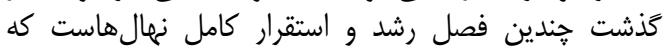

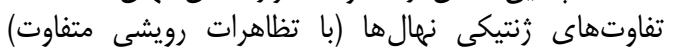

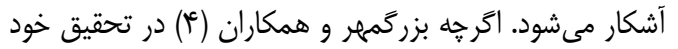

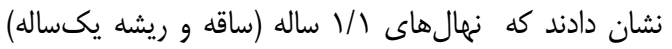

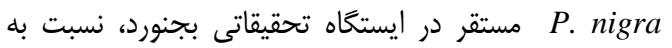

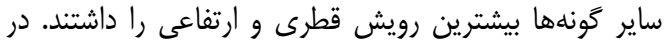

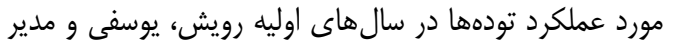

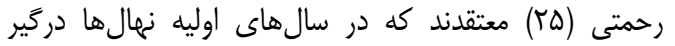

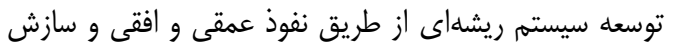

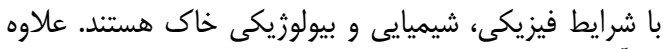

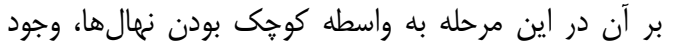

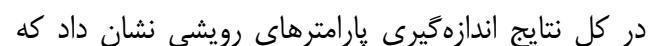

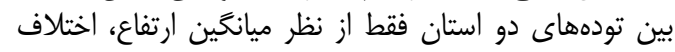

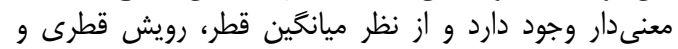

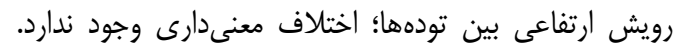

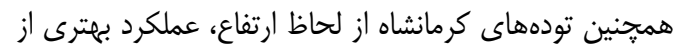

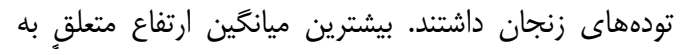

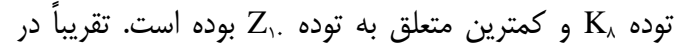

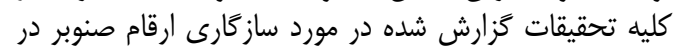

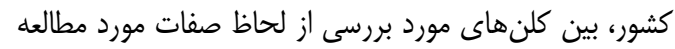

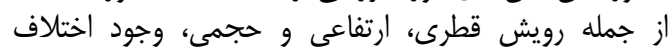

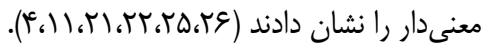

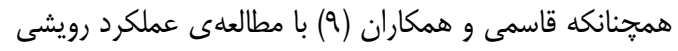
و فنولوزى كلنهاى مختلف صنوبر در ايستخاه تحقيقاتى البرز كرج، نشان دادند كه كلنهاى

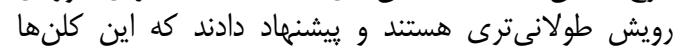

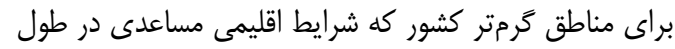

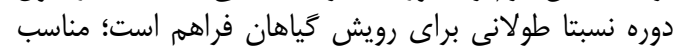

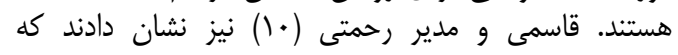

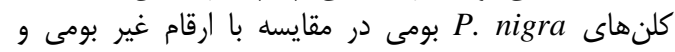

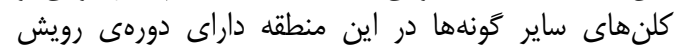

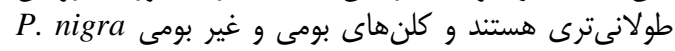

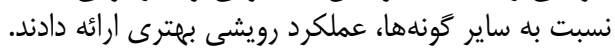

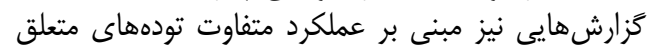

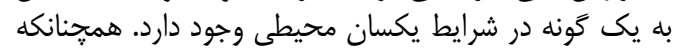

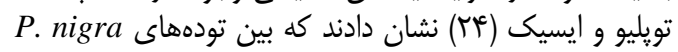

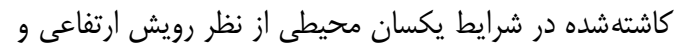

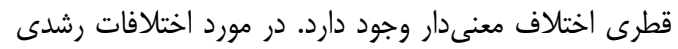

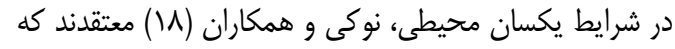

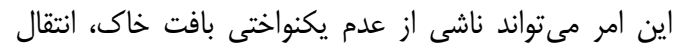

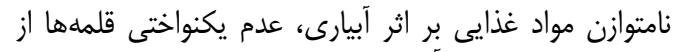

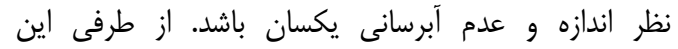

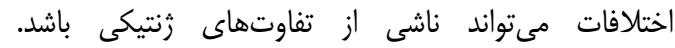

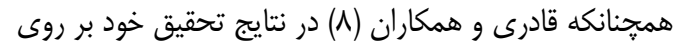

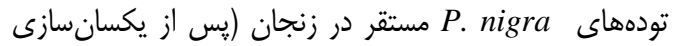


خرداد)، مرحله رشد سريع (خرداد تا شهريور) و مرحله رشد

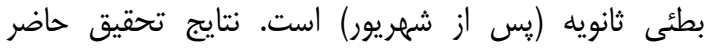

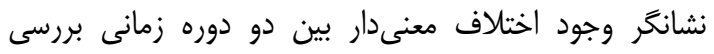

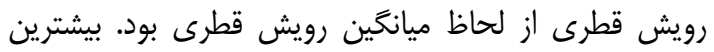

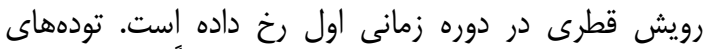

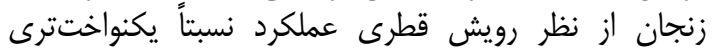

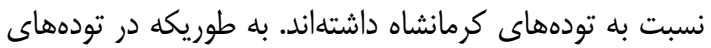

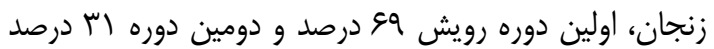
رويش كل را شامل مى شود. ولى دور دور تودههای دور كرمانشاه، اولين

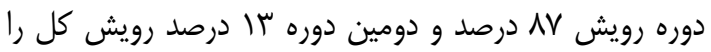

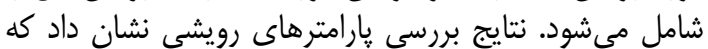

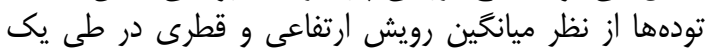
فصل رشد اختلاف معنى دارى ندارند.

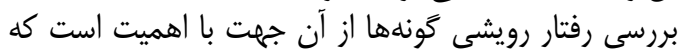

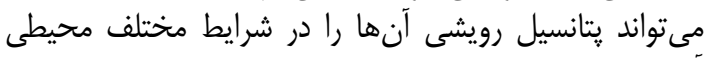

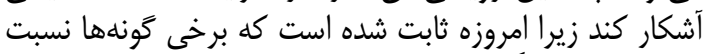

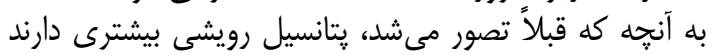

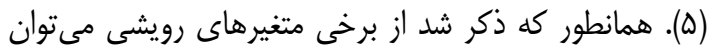

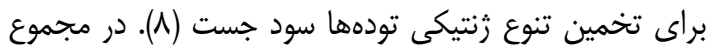

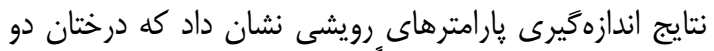

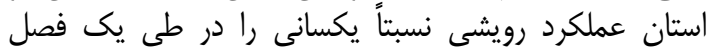
رشد ارائه دادند.

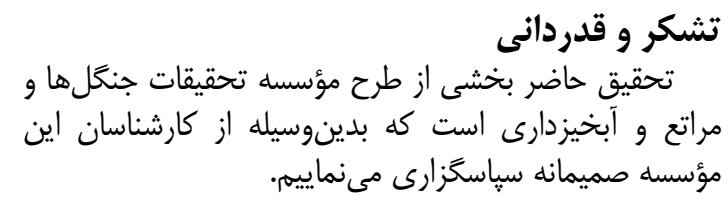

فضاى خالى قابل توجه بين آنها و رطوبت ناشى از آبيارى؛

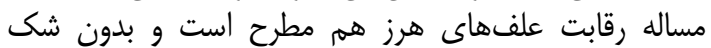

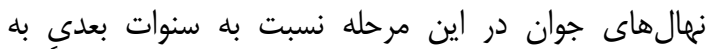

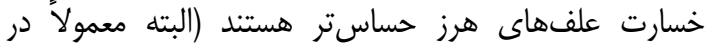
آزمايشهاى مزرعهاى، مثل تحقيق حاضر، علفهای عاى هاى هرز

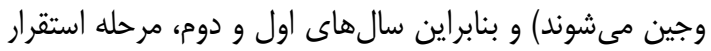

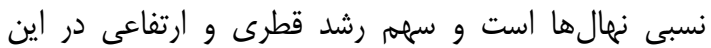

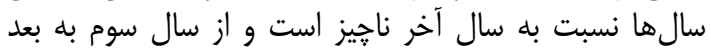

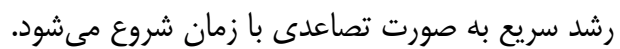

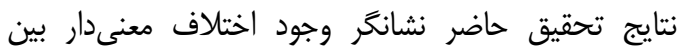

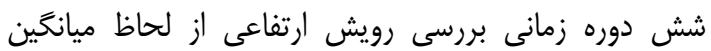

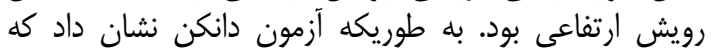

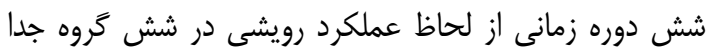

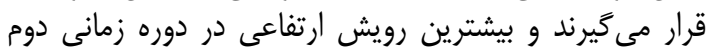

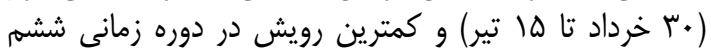

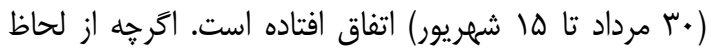

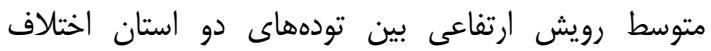

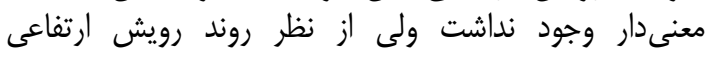

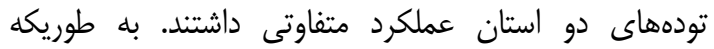

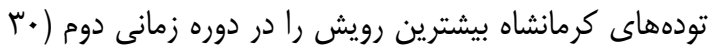

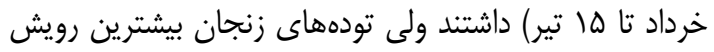

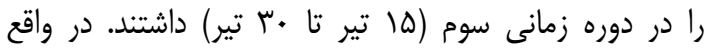

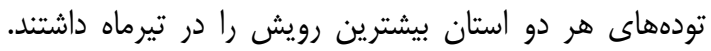

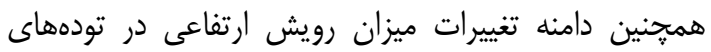

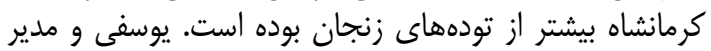

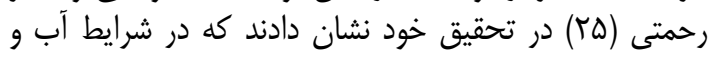

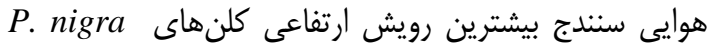
Kamyaran

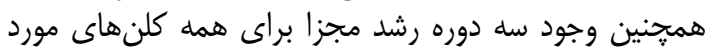
بررسى را نشان دادند كه شامل مرحله رشد بـ بطئى اوليه (قبل از

منابع

1. Alimohamadi, A., F. Asadi and R. Tabaie Aghdaei. 2015. Evaluation of growth and morphological parameters in two poplar species (P.nigra L. \& P. alba L.) to tree growth reveal traits related to productivity (case study in Kermanshah, Zanjan and Esfahan provinces). Ecology of Iranian Forest, 3(5): 31-41 (In Persian).

2. Alimohamadi, A. 2008. Investigation on genetic diversity in Populus nigra stands using molecular and morphological markers. Ph.D. Thesis, Science \& Research Branch of Islamic Azad University, Tehran, Iran. 152 pp. (In Persian)

3. Asadi, F., F. Noori and B. Yousefi. 2015. Growth variations in poplar (Populus nigra L.) plantations in riverbanks of Kermanshah Province. Iranian Journal of Forest and Poplar Research, 23(2): 209-221 (In Persian).

4. Bozorg Mehr, A., A.R. Modir Rahmati, R. Ghasemi, A. Kalantari and S. Safari. 2008. Comparative of native and exotic poplar clones in north Khorasan province. Proceeding of the Second National Congress on Poplar and Potential use in Poplar Plantation, 288-300 pp., Tehran, Iran (In Persian).

5. Christerson, L. 2006. Biomass production of intensively grown poplars in the southernmost part of Sweden: Observations of characters, traits and growth potential. Biomass and Bioenergy, 30(6): 497508.

6. Fo Perry, C.H., R.C. Miller and K.N. Brooks. 2001. Impacts of short-rotation hybrid poplar plantations on regional water yield. Forest Ecological Management, 143(1-3): 143-151.

7. Fortier, J., D. Gagnon, B. Truax, and F. Lambert. 2010. Nutrient accumulation and carbon sequestration in 6-year-old hybrid poplars in multi clonal agricultural riparian buffer strip. Agriculture Ecosystems and Environment, 137(3-4): 276-287. 
$M$ ارزيابى عملكرد رويشى درختان تبريزى (Populus nigra) بومى استانهاى كرمانشاه و زنجان .

8. Ghaderi, T.H., A.R. Modir Rahmati, A. Hemati, M.G. Nabii, P. Moradi and A. Jafari. An investigation on heritability and relationships between the traits of thirteen clones of close canopy poplars in the condition of Zanjan. Proceeding of the Second National Congress on Poplar and Potential Use in Poplar Plantation, 261-268 pp., Tehran, Iran (In Persian).

9. Ghasemi, R., A. Jalili, M. Akbarinia and A.R. Modir Rahmati. 2001. Investigation on phenolory of different poplar clones in collection at Karaj research station during 1997 \& 1998 period. Iranian Journal of Forest and Poplar Research, 6(1): 63-94 (In Persian).

10. Ghasemi, R. and A.R. Modir Rahmati. 2003. Investigation on adaptability and wood production of different poplar clones (closed crown) in Karaj City. Iranian Journal of Forest and Poplar Research, 11(3): 359-390 (In Persian).

11. Gholami, G.H., A.R. Modir Rahmati and R. Ghasemi. 2000. Some characteristics of poplar saplings (1/1) in experimental nurseries of Golestan province. Iranian Journal of Forest and Poplar Research, 18(2): 177-189 (In Persian).

12. Gholami, G.H., A. Modir Rahmati, R. Ghasemi and S.Z. Mirkazemi. 2014. Adaptation and wood production of different closed-crown poplar clones in Gorgan region. Iranian Journal of Forest and Poplar Research, 22(3): 473-484. (In Persian)

13. Isik, F and F. Toplu. 2004. Variation in juvenile traits of natural black poplar (Popolus nigra L.). clones in Turkey. New Forest, 27(2): 175-187.

14. Joshi, R.P. and N.P. Singh. 1996. Path coefficient analysis in Poplar (Populus deltoides). Indian Journal of Environment and Toxicology, 6: 95-97.

15. Karimi,G., A.R. Modir Rahmati and R. Rahmani. 2004. Test the final stage adaptation of different poplar clones at Safrabasteh, The final report of the research project, Research Institute of Forests and Rangelands.46 pp (In Persian).

16. Lotfian, H., S.F. Ziaii Ziabari, A.R. Modir Rahmati, S. Ghaicy and A. Hammati. 1984. A simplify guide for research method in Populus species in Iran. Institute of Forests and Rangelands, 37 pp (In Persian).

17. Micheal, D.A., J.G. Isebrands, D.I. Dickmann and N.D. Nelson. 1988. Growth and development during the establishment year of two Populus clones with contrasting morphology and phenology Tree Physiol, 4(2): 139-152.

18. Nooki, Y., A.R. Modir Rahmati, A. Hemmati and A. Aimani. 2008. A survey on year old seedling of different poplar clones at experimental nursery of Ardabil. Proceeding of the Second National Congress on Poplar and Potential Use in Poplar Plantation, 309-319 pp., Tehran, Iran (In Persian).

19. Rostamikia, Y., A.R. Modir Rahmati and M. Tabari Kouchaksaraei. 2017. Growth characteristics of native and exotic clones of poplar in Ardabil nursery. Journal of Plant Research, 30(2): 454-466 (In Persian).

20. Sadeghi, A., A. Salehi and S.A. Mousavi Koupar. 2015. Effect of poplar monoculture and poplar whit peanut as an agroforestry cultivation on soil chemical propertie. Ecology of Iranian Forest, 3(6): 28-35 (In Persian).

21. Soheili Esfahani, S. and A. Fallah. 2014. The study of qualitative, quantitative characteristics of traditional poplar (Populus alba and Populus nigra) plantations in western Esfahan province. Journal of Forest and Wood Product, 67(2): 233-244 (In Persian).

22. Talebi, M., A.R. Modir Rahmati, Y. Iranmanesh and H. Jahanbazi. 2008. The survey on six exotic poplar vegetation in mass plantation in Chaharmahal and Bakhtiary province. Proceeding of the Second National Congress on Poplar and Potential Use in Poplar Plantation, 78-87 pp., Tehran, Iran (In Persian).

23. Tavousi Rad, F., A. Ghamari Zare, H. Mirzaie Nodoushan and M. Usefifard. 2017. Evaluation of poplar inter-specific progenies based on their morphologic and micro morphologic traits. Iranian Journal of Forest and Poplar Research, 24(4): 675-686 (In Persian).

24. Toplu, F. and F. Isik. 1999. Nursery performances of black poplar clones in the southest Turkey. International symposium II, Program with abstracts, 13-17 pp., Orlean, France.

25. Yousefi, B. and A.R. Modir Rahmati. 2011. Survey on adaptation and wood yield of Populus nigra clones in comparative populetum of Sanandaj (final stage). Iranian Journal of Forest and Poplar Research, 19(3): 293-294 (In Persian).

26. Yousefi, B. and A.R. Modir Rahmati. 2004. Height growth behavior of Poplar clones in Kurdistan. Iranian Journal of Forest and Poplar Research, 13(4): 533-556 (In Persian). 


\title{
Study of Growth Function of Black Poplar (Populus Nigra) With Kermanshah and Zanjan Origin in Karaj Research Station
}

\section{Afrooz Alimohamadi ${ }^{1}$ and Farhad Asadi $^{2}$}

\author{
1- Corresponding author, Department of Agriculture and Natural Resources, Payame Noor University (PNU), Tehran, \\ IRAN, (Corresponding Author: afrooz.alimohamadi@pnu.ac.ir) \\ 2- Research Division of Natural Resources, Mazandaran Agricultural and Natural Resources Research Center, \\ AREEO, Sari, IRAN \\ Receive: September 7, 2018 \\ Accepted: September 30, 2018
}

\begin{abstract}
In order to evaluate the growth function of Populus nigra trees under homogeneous conditions, a total of 24 individuals of $P$. nigra (3 individuals per stand) were sampled from 8 artificial stands in Kermanshah province and 12 individuals of $P$. nigra ( 3 individuals per stand) were sampled from 4 artificial stands in Zanjan province. In order to assimilate the environmental conditions, seedlings were planted under randomized complete blocks design with 3 replications in Karadj area. In growing season, height growths were measured in 6 periods and diameter growths were measured in 2 periods. The results showed no significant differences between the stands in term of height and diameter growths but there were significant differences between periods in terms of height and diameter growths. In total, the results of growth parameters measuring in $P$. nigra stands showed that the trees of the two provinces presented a fairly similar growth function during a growing season.
\end{abstract}

Keywords: Diameter Growth, Height Growth, Kermanshah and Zanjan Provinces and Populus Nigra 